\title{
Phase Transition of RN-AdS Black Hole with Fixed Electric Charge and Topological Charge
}

\author{
Shan-Quan Lan (i) \\ Department of Physics, Lingnan Normal University, Zhanjiang, 524048, Guangdong, China \\ Correspondence should be addressed to Shan-Quan Lan; shanquanlan@126.com
}

Received 5 September 2018; Revised 28 October 2018; Accepted 25 November 2018; Published 11 December 2018

Academic Editor: Elias C. Vagenas

Copyright (C) 2018 Shan-Quan Lan. This is an open access article distributed under the Creative Commons Attribution License, which permits unrestricted use, distribution, and reproduction in any medium, provided the original work is properly cited. The publication of this article was funded by SCOAP S $^{3}$

\begin{abstract}
Phase transition of RN-AdS black hole is investigated from a new perspective. Not only is the cosmological constant treated as pressure but also the spatial curvature of black hole is treated as topological charge $\epsilon$. We obtain the extended thermodynamic first law from which the mass is naturally viewed as enthalpy rather than internal energy. In canonical ensemble with fixed topological charge and electric charge $Q$, interesting van der Waals like oscillatory behavior in $T-S$ and $P-V$ graphs and swallow tail behavior in $G-T$ and $G-P$ graphs is observed. By applying the Maxwell equal area law and analysing the Gibbs free energy, we obtain analytical phase transition coexistence curves which are consistent with each other. The phase diagram is four dimensional with $T, P, Q, \epsilon$.
\end{abstract}

\section{Introduction}

Black hole is a simple object which can be described by only a few physical quantities, such as mass, charge, and angular momentum, while it is also a complicate thermodynamic system. Since the discovery of black hole's entropy [1], the four-thermodynamic law [2], and the Hawking radiation [3] in 1970s, thermodynamic of black hole has become an interesting and challenging topic. Especially, in the anti-de Sitter (AdS) space, there exists Hawking-Page phase transition between stable large black hole and thermal gas [4]. Due to the AdS/CFT correspondence [5-7], the Hawking-Page phase transition is explained as the confinement/deconfinement phase transition of a gauge field [8].

When the AdS black hole is electrically charged, its thermodynamic properties become more rich. In the canonical ensemble with fixed electric charge, there is a first-order phase transition between small and large black holes [9-12]. Increasing the temperature, the phase transition coexistence curve ends at the critical point, where the first-order phase transition becomes a second-order one. In the grand canonical ensemble with fixed temperature, there is also a critical temperature. Below the critical temperature, $\Phi(Q)$ is a singlevalued function, where $Q$ is electric charge and $\Phi$ is the conjugate potential. Above the critical temperature, $\Phi(Q)$ is a multivalued function with phase transitions [12]. The phase transition behavior of AdS black hole is reminiscent to the liquid-gas phase transition in a van der Waals system.

Viewing the cosmological constant as a dynamical pressure and the black hole volume as its conjugate quantity [13], the analogy of charged AdS black hole as a van der Waals system has been further enhanced in Ref. [14]. Both the systems share the same oscillatory behavior in pressurevolume graph and swallow tail behavior in Gibbs free energytemperature (pressure) graph. What's more, they have very similar phase diagrams and have exactly the same critical exponents. The phase transition property is also investigated in temperature-entropy graph [15]. Later, this analogy has been generalized to different AdS black holes, such as rotating black holes, higher dimensional black holes, Gauss-Bonnet black holes, $f(R)$ black holes, black holes with scalar hair, etc [15-57], where more interesting phenomena are found.

Recently, the spatial curvature of electrically charged AdS black hole is viewed as variable and treated as topological 
charge $[58,59]$ in Einstein-Maxwell's gravity and LovelockMaxwell theory. The authors found that the topological charge naturally arose in holography. What is more, together with all other known charges (electric charge, mass, and entropy), they satisfy an extended first law and the GibbsDuhem-like relation as a completeness. In our last paper [60], when the cosmological constant is not viewed as variable, we find a van der Waals type but new phase transition relating to the topological charge, while in this paper, we will treat both the cosmological constant and the spatial curvature as variables, then following one of their methods to derive the extended first law, from which one can see the cosmological constant is naturally viewed as pressure and the mass is viewed as enthalpy. Based on the extended first law, the black hole's phase transition property will be investigated in canonical ensemble with fixed electric charge and topological charge.

This paper is organized as follows. In Section 2, following the method in Ref. [59], we will derive the extended first law in d dimensional space-time. In Section 3, by analysing the specific heat, the phase transition of AdS black hole in 4dimensional space-time is studied and the critical point is determined. In Section 4, the van der Waals like oscillatory behavior is observed in both $T-S$ and $P-V$ graphs. Then we use the Maxwell equal area law to obtain the phase transition coexistence curve. In Section 5, the van der Waals like swallow tail behavior is observed in $G-T$ and $G-P$ graphs, then we will obtain the phase transition coexistence curve by analysing the gibbs free energy. Finally, we summarize and discuss our results in Section 6.

\section{The Extended Thermodynamic First Law}

The d dimensional space-time AdS black hole solutions with maximal symmetry in the Einstein-Maxwell theory are

$$
d s^{2}=\frac{d r^{2}}{f(r)}-f(r) d t^{2}+r^{2} d \Omega_{d-2}^{(k) 2},
$$

where

$$
\begin{aligned}
f(r) & =k+\frac{r^{2}}{l^{2}}-\frac{m}{r^{d-3}}+\frac{q^{2}}{r^{2 d-6}}, \\
d \Omega_{d-2}^{(k) 2} & =\widehat{g}_{i j}^{(k)}(x) d x^{i} d x^{j}, \\
A & =-\frac{\sqrt{d-2} q}{\sqrt{2(d-3)} r^{d-3}} d t .
\end{aligned}
$$

$m, q, l$ are related to the ADM mass $M$, electric charge $Q$, and cosmological constant $\Lambda$ by

$$
\begin{aligned}
M & =\frac{(d-2) \Omega_{d-2}^{(k)}}{16 \pi} m, \\
Q & =\sqrt{2(d-2)(d-3)}\left(\frac{\Omega_{d-2}^{(k)}}{8 \pi}\right) q, \\
\Lambda & =-\frac{(d-1)(d-2)}{2 l^{2}},
\end{aligned}
$$

and $\Omega_{d-2}^{(k)}$ is the volume of the "unit" sphere, plane, or hyperbola, and $k$ stands for the spatial curvature of the black hole. Under suitable compactifications for $k \leq 0$, we assume that the volume of the unit space is a constant $\Omega_{d-2}=\Omega_{d-2}^{(k=1)}$ hereafter $[58,59]$.

Following [59], the first law of thermodynamics can be derived. As the first law of thermodynamics is about the differential relation of every physical quantity, one can first find an equation containing these physical quantities and then differentiate it to obtain the first law of thermodynamics. Considering an equipotential surface $f(r)=c$ with fixed $c$ (here set $c=0$ ), we variate both sides of the equation and obtain

$$
\begin{aligned}
d f\left(r_{+}, k, m, q\right)= & \frac{\partial f}{\partial r_{+}} d r_{+}+\frac{\partial f}{\partial k} d k+\frac{\partial f}{\partial\left(1 / l^{2}\right)} d \frac{1}{l^{2}} \\
& +\frac{\partial f}{\partial m} d m+\frac{\partial f}{\partial q} d q=0,
\end{aligned}
$$

where $r_{+}$is the radius of event horizon. Noting

$$
\begin{aligned}
\partial_{r_{+}} f & =4 \pi T, \\
\partial_{k} f & =1, \\
\partial_{1 / l^{2}} f & =r_{+}^{2}, \\
\partial_{m} f & =-\frac{1}{r_{+}^{d-3}}, \\
\partial_{q} f & =\frac{2 q}{r_{+}^{2 d-6}},
\end{aligned}
$$

we obtain

$$
d m=\frac{4 \pi T}{d-2} d r_{+}^{d-2}+r_{+}^{d-3} d k+r_{+}^{d-1} d \frac{1}{l^{2}}+\frac{2 q}{r_{+}^{d-3}} d q
$$

Multiplying both sides with an constant factor ( $d$ 2) $\Omega_{d-2} / 16 \pi$, the above equation becomes

$$
\begin{aligned}
d M= & T d S+\frac{(d-2) \Omega_{d-2}}{16 \pi} r_{+}^{d-3} d k \\
& +\frac{(d-2) \Omega_{d-2}}{16 \pi} r_{+}^{d-1} d \frac{1}{l^{2}}+\Phi d Q
\end{aligned}
$$

where $T=\partial_{r_{+}} f / 4 \pi$ is the temperature, $S=\left(\Omega_{d-2} / 4\right) r_{+}^{d-2}$ is the entropy, and $\Phi=\sqrt{(d-2) / 2(d-3)}\left(q / r_{+}^{d-3}\right)$ is the electric potential. If we introduce a new "charge" as in $[58,59]$

$$
\epsilon=\Omega_{d-2} k^{(d-2) / 2},
$$

then its conjugate potential is obtained as $\omega=(1 /$ $8 \pi) k^{(4-d) / 2} r_{+}^{d-3}$. If we define the black hole volume as $V=$ $\left(\Omega_{d-2} /(d-1)\right) r_{+}^{d-1}$, then its conjugate pressure is naturally arisen as $P=(d-1)(d-2) / 16 \pi l^{2}$, and the black hole mass 
is naturally viewed as enthalpy instead of energy. Finally, the extended first law is obtained as

$$
d M=T d S+\omega d \epsilon+V d P+\Phi d Q
$$

\section{The Specific Heat and Phase Transition}

Hereafter, the investigation will be limited in $d=4$ dimensional space-time and in canonical ensemble with fixed electric charge and topological charge, leaving other situations for further study. First of all, we would like to analyse the behavior of the specific heat and the related possible phase transition phenomena. The first law can be rewritten in terms of energy $E=M-P V$,

$$
d E=T d S-P d V \text {. }
$$

So the isobaric specific heat can be written as

$$
\begin{aligned}
C_{P, \mathrm{Q}, \epsilon} & =T\left(\frac{\partial S}{\partial T}\right)_{P, \mathrm{Q}, \epsilon} \\
& =\frac{2 \pi r_{+}^{2}\left(32 \pi^{2} P r_{+}^{4}+\epsilon r_{+}^{2}-4 \pi Q^{2}\right)}{32 \pi^{2} P r_{+}^{4}-\epsilon r_{+}^{2}+12 \pi Q^{2}}
\end{aligned}
$$

Since we are in canonical ensemble, $C_{P, \mathrm{Q}, \epsilon}$ can be abbreviated as $C_{P}$. From the denominator, we can conclude the following:

(1) When $P<\epsilon^{2} / 1536 \pi^{3} Q^{2}, C_{P}$ has two diverge points at

$$
r_{+(1,2)}=\frac{1}{8 \pi} \sqrt{\frac{\epsilon \pm \sqrt{\epsilon^{2}-1536(\pi)^{3} Q^{2} P}}{P}},
$$

which signals a phase transition.

(2) When $P=P_{c}=\epsilon^{2} / 1536 \pi^{3} Q^{2}$, the two diverge points of $C_{P}$ merge into one at

$$
r_{+}=r_{c}=2 \sqrt{\frac{6 \pi}{\epsilon}} Q
$$

which is the phase transition critical point. The critical temperature $T_{c}=\epsilon^{3 / 2} / 24 \sqrt{6}(\pi)^{5 / 2} Q$.

(3) When $P>\epsilon^{2} / 1536 \pi^{3} Q^{2}, C_{P}$ is always larger than zero, so there is no phase transition.

Comparing with the van der Waals equation, the specific volume is defined as [14]

$$
v=2 r_{+} .
$$

At the critical point, we obtain an interesting relation

$$
\frac{P_{c} v_{c}}{T_{c}}=\frac{3}{8}
$$

which is exactly the same as for the van der Waals fluid and $\mathrm{RN}$-AdS black holes. Note that this number which seems to be universal does not depend on the topological charge or electric charge.
All the physical quantities can be rescaled by those at the critical point. Defining

$$
\begin{aligned}
r_{+} & =\tilde{r} r_{c}, \\
P & =\widetilde{P} P_{c}
\end{aligned}
$$

the isobaric specific heat becomes

$$
C_{P}=\frac{16 \pi^{2} Q^{2}}{\epsilon} \frac{\widetilde{r}^{2}\left(3 \widetilde{P} \widetilde{r}^{4}+6 \widetilde{r}^{2}-1\right)}{\widetilde{P} \widetilde{r}^{4}-2 \widetilde{r}^{2}+1} \equiv \frac{16 \pi^{2} Q^{2}}{\epsilon} \widetilde{C}_{\widetilde{P}}
$$

The behaviors of the rescaled specific heat $\widetilde{C}_{\widetilde{P}}$ for the cases $P<P_{c}, P=P_{c}$, and $P>P_{c}$ are shown in Figure 1. The curve of specific heat for $P<P_{c}$ has two divergent points which divide the region into three parts. Both the large radius region and the small radius region are thermodynamically stable with positive specific heat, while the medium radius region is unstable with negative specific heat. So there is a phase transition which takes place between small black hole and large black hole. The curve of specific heat for $P=P_{c}$ has only one divergent point and always positive denoting that $\epsilon_{c}$ is exactly the critical point. The curve of specific heat for $P>P_{c}$ has no divergent point and is always positive, implying that the black holes are stable and no phase transition will take place. This behavior of specific heat is very similar to that of the liquid-gas var der Waals system.

\section{Oscillatory Behavior in $T-S$ and $P-V$ Graphs: Phase Transition Coexistence Curve}

In the last section, we have determined the critical point and found a phase transition when $P \leq P_{c}$. In this section and in the next section, we will derive the analytical phase transition coexistence curve by using different methods.

4.1. Maxwell Equal Area Law in T - S Graph and Phase Transition Coexistence Curve. The temperature and entropy are

$$
\begin{aligned}
T & =\frac{f^{\prime}\left(r_{+}\right)}{4 \pi}=\frac{1}{4 \pi r_{+}}\left(k-\frac{q^{2}}{r_{+}^{2}}+\frac{3 r_{+}^{2}}{l^{2}}\right) \\
& =\frac{\epsilon}{16 \pi^{2} r_{+}}-\frac{Q^{2}}{4 \pi r_{+}^{3}}+2 P r_{+}, \\
S & =\pi r_{+}^{2},
\end{aligned}
$$

which can be rescaled by (16) to be

$$
\begin{aligned}
& T=\frac{3 \widetilde{P} \widetilde{r}^{4}+6 \widetilde{r}^{2}-1}{8 \widetilde{r}^{3}} \frac{\epsilon^{3 / 2}}{24 \sqrt{6} \pi^{5 / 2} Q}=\widetilde{T} T_{c}, \\
& S=\widetilde{r}^{2} \frac{24 \pi^{2} Q^{2}}{\epsilon}=\widetilde{S} S_{c},
\end{aligned}
$$

here $S_{c} \equiv \pi r_{c}^{2}=24 \pi^{2} Q^{2} / \epsilon$. Thus we obtain

$$
\widetilde{T}=\frac{3 \widetilde{P} \widetilde{S}^{2}+6 \widetilde{S}-1}{8 \widetilde{S}^{3 / 2}} .
$$



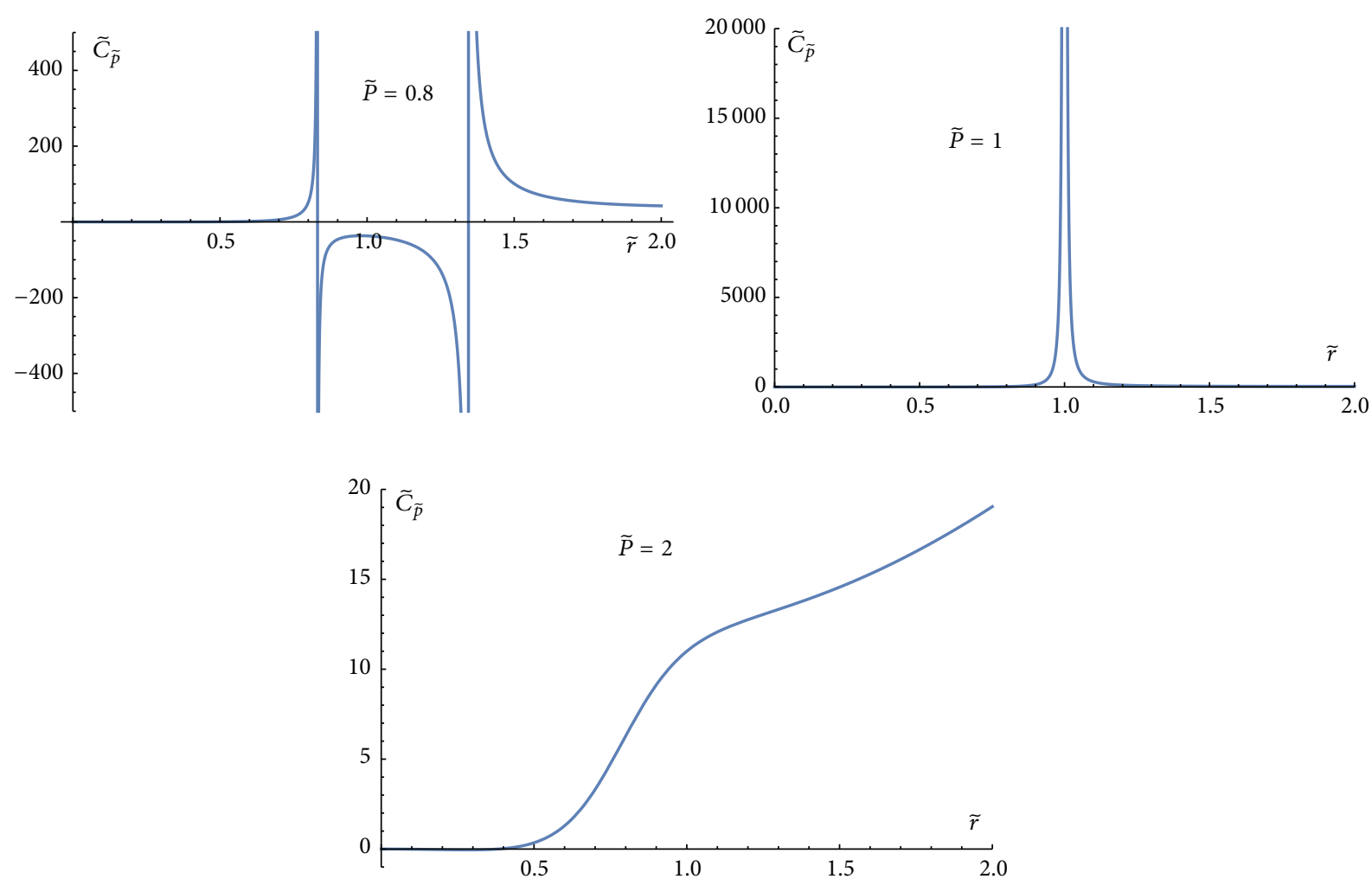

Figure 1: The specific heat $\widetilde{C}_{\widetilde{P}}$ vs. $\widetilde{r}$ for $\widetilde{P}=0.8<\widetilde{P}_{c}$ which has two divergent points, $\widetilde{P}=1=\widetilde{P}_{c}$ which has only one divergent point and $\widetilde{P}=2>\widetilde{P}_{c}$ which has no divergent point.

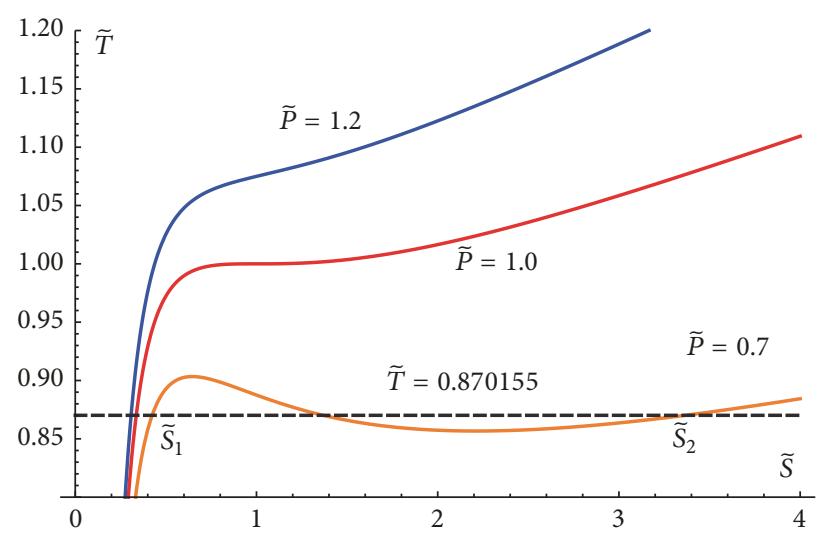

Figure $2: \widetilde{T}$ vs. $\widetilde{S}$ for $\widetilde{P}=0.7,1.0,1.2$. The phase transition take place for $\widetilde{P} \leq 1.0$. The dashed black line $\widetilde{T}=0.870155$ equally separates the oscillatory part. According to Maxwell's equal area law, the phase transition point is $(\widetilde{T}=0.870155, \widetilde{P}=0.7)$.

From the above equation, we can plot the curve $\widetilde{T}(\widetilde{S})$ for different $\widetilde{P}$ in Figure 2. One can see that, for pressure $\widetilde{P} \leq$ 1.0 , temperature $\widetilde{T}(\widetilde{S})$ curves show interesting var der Waals system's oscillatory behavior which denote the existence of phase transition. The oscillatory part needs to be replaced by an isobar (denote as $\widetilde{T}^{*}$ ) such that the areas above and below it are equal to each other. This treatment obeys Maxwell's equal area law. In what follows, we will analytically determine this isobar $\widetilde{T}^{*}$ for different $\widetilde{P}$.
Maxwell's equal area law is manifest as

$$
\begin{aligned}
\widetilde{T}^{*}\left(\widetilde{S}_{2}-\widetilde{S}_{1}\right)= & \int_{\widetilde{S}_{1}}^{\widetilde{S}_{2}} \widetilde{T}(\widetilde{S}, \widetilde{P}) d \widetilde{S} \\
= & \frac{\widetilde{P}}{4}\left(\widetilde{S}_{2}^{3 / 2}-\widetilde{S}_{1}^{3 / 2}\right)+\frac{3}{2}\left(\widetilde{S}_{2}^{1 / 2}-\widetilde{S}_{1}^{1 / 2}\right) \\
& +\frac{1}{4}\left(\widetilde{S}_{2}^{-1 / 2}-\widetilde{S}_{1}^{-1 / 2}\right) .
\end{aligned}
$$



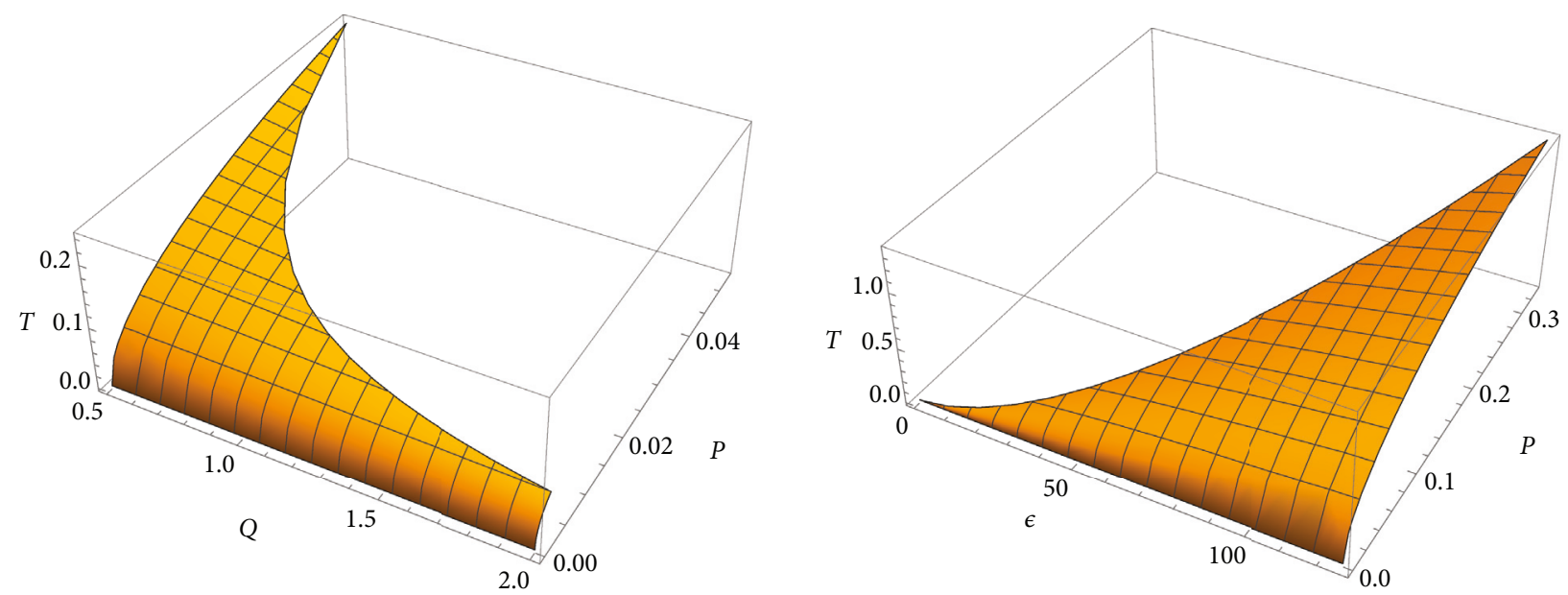

FIGURE 3: The phase transition coexistence curves for $\epsilon=8 \pi$ (left) and $Q=1$ (right). The end points are the critical points.

At points $\left(\widetilde{S}_{1}, \widetilde{T}^{*}\right)$ and $\left(\widetilde{S}_{2}, \widetilde{T}^{*}\right)$, we have two equations

$$
\begin{aligned}
& \widetilde{T}^{*}=\frac{3 \widetilde{P} \widetilde{S}_{1}^{2}+6 \widetilde{S}_{1}-1}{8 \widetilde{S}_{1}^{3 / 2}}, \\
& \widetilde{T}^{*}=\frac{3 \widetilde{P} \widetilde{S}_{2}^{2}+6 \widetilde{S}_{2}-1}{8 \widetilde{S}_{2}^{3 / 2}} .
\end{aligned}
$$

The above three equations can be solved as

$$
\begin{aligned}
& \widetilde{S}_{1}=\frac{(\sqrt{3-\sqrt{\widetilde{P}}}-\sqrt{3-3 \sqrt{\widetilde{P}}})^{2}}{2 \widetilde{P}}, \\
& \widetilde{S}_{2}=\frac{(\sqrt{3-\sqrt{\widetilde{P}}}+\sqrt{3-3 \sqrt{\widetilde{P}}})^{2}}{2 \widetilde{P}}, \\
& \widetilde{T}^{*}=\sqrt{\frac{\widetilde{P}(3-\sqrt{\widetilde{P}})}{2}} .
\end{aligned}
$$

The last equation $\widetilde{T}^{*}(\widetilde{P})$ is the rescaled phase transition coexistence curve. Then we can make a backward rescale to obtain the phase transition coexistence curve,

$$
T=\frac{\sqrt{2 P\left(3 \epsilon-16 \sqrt{6} \pi^{3 / 2} Q \sqrt{P}\right)}}{3 \pi} .
$$

Note that the phase diagram is four dimensional $(T, P, Q, \epsilon)$. The condition for the phase transition is that $\epsilon>$ $16 \sqrt{6} \pi^{3 / 2} Q \sqrt{P} / 3$. When the topological charge $\epsilon=0$, there will be no phase transition. While when the electric charge $Q \rightarrow 0(Q>0)$, there will be phase transition with the critical temperature $T_{c} \longrightarrow \infty$ and pressure $P_{c} \longrightarrow$ $\infty$. The detailed dependence of the phase transition on the topological charge can be seen in (24) and in right graph of Figure 3.
The phase transition coexistence curves are plotted in Figure 3 for $\epsilon=8 \pi$ (left) and $Q=1$ (right). The end points in the graphs are the critical points. With fixed $\epsilon$ and $Q$, the phase transition coexistence curve $T(P)$ is reminiscent of the var der Waals system's liquid-gas phase transition coexistence curve.

4.2. Maxwell Equal Area Law in P - V Graph and Phase Transition Coexistence Curve. The pressure and volume are

$$
\begin{aligned}
& P=\frac{T}{2 r_{+}}-\frac{\epsilon}{32 \pi^{2} r_{+}^{2}}+\frac{Q^{2}}{8 \pi r_{+}^{4}}, \\
& V=\frac{4}{3} \pi r_{+}^{3},
\end{aligned}
$$

which can be rescaled to be

$$
\begin{aligned}
& P=\left(\frac{8 \widetilde{T}}{3 \widetilde{r}}-\frac{2}{\widetilde{r}^{2}}+\frac{1}{3 \widetilde{r}^{4}}\right) \frac{\epsilon^{2}}{1536 \pi^{3} Q^{2}}=\widetilde{P} P_{c}, \\
& V=\widetilde{r}^{3} 64 \sqrt{6} \pi^{5 / 2}\left(\frac{Q^{2}}{\epsilon}\right)^{3 / 2}=\widetilde{V} V_{c} .
\end{aligned}
$$

Thus we obtain

$$
\widetilde{P}=\frac{8 \widetilde{T}}{3} \widetilde{V}^{-1 / 3}-2 \widetilde{V}^{-2 / 3}+\frac{1}{3} \widetilde{V}^{-4 / 3}
$$

From the above equation, we can plot the curve $\widetilde{P}(\widetilde{V})$ for different $\widetilde{T}$ in Figure 4. One can see that for temperature $\widetilde{T} \leq 1.0$, pressure $\widetilde{P}(\widetilde{V})$ curves show interesting var der Waals system's oscillatory behavior which corresponds to the phase transition. Similarly, the oscillatory part needs to be replaced by an isobar (denote as $\widetilde{P}^{*}$ ) such that the areas above and below it are equal to each other. This treatment follows Maxwell's equal area law. The analytical phase transition curve is derived as follows. 


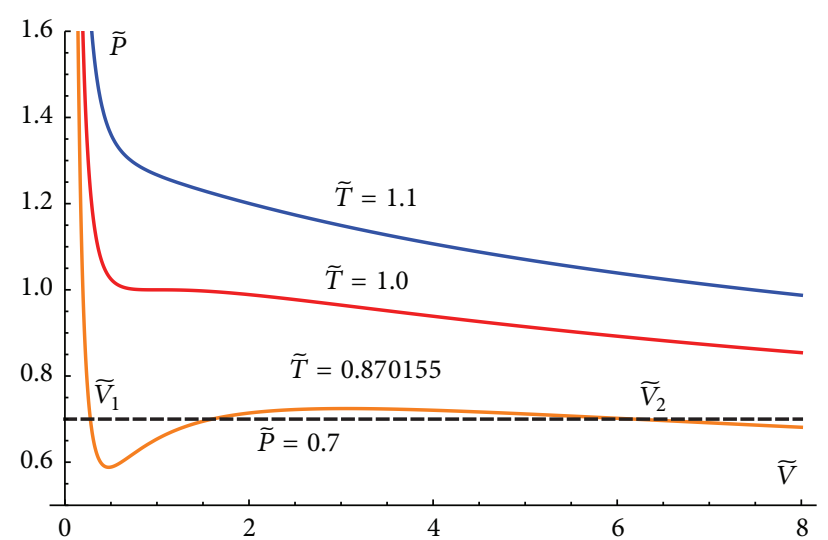

Figure 4: $\widetilde{P}$ vs. $\widetilde{V}$ for $\widetilde{T}=0.870155,1.0,1.1$. The phase transition takes place for $\widetilde{T} \leq 1.0$. The dashed black line $\widetilde{P}=0.7$ equally separates the oscillatory part. According to Maxwell's equal area law, the phase transition point is $(\widetilde{T}=0.870155, \widetilde{P}=0.7)$.

Maxwell's equal area law is manifest as

$$
\begin{aligned}
\widetilde{P}^{*}\left(\widetilde{V}_{2}-\widetilde{V}_{1}\right)= & \int_{\widetilde{V}_{1}}^{\widetilde{V}_{2}} \widetilde{P}(\widetilde{V}, \widetilde{T}) d \widetilde{V} \\
= & 4 \widetilde{T}\left(\widetilde{V}_{2}^{2 / 3}-\widetilde{V}_{1}^{2 / 3}\right)-6\left(\widetilde{V}_{2}^{1 / 3}-\widetilde{V}_{1}^{1 / 3}\right) \\
& -\frac{1}{3}\left(\widetilde{V}_{2}^{-1 / 3}-\widetilde{V}_{1}^{-1 / 3}\right) .
\end{aligned}
$$

At points $\left(\widetilde{V}_{1}, \widetilde{P}^{*}\right)$ and $\left(\widetilde{V}_{2}, \widetilde{P}^{*}\right)$, we have two equations

$$
\begin{aligned}
& \widetilde{P}^{*}=\frac{8 \widetilde{T}}{3} \widetilde{V}_{1}^{-1 / 3}-2 \widetilde{V}_{1}^{-2 / 3}+\frac{1}{3} \widetilde{V}_{1}^{-4 / 3}, \\
& \widetilde{P}^{*}=\frac{8 \widetilde{T}}{3} \widetilde{V}_{2}^{-1 / 3}-2 \widetilde{V}_{2}^{-2 / 3}+\frac{1}{3} \widetilde{V}_{2}^{-4 / 3} .
\end{aligned}
$$

The above three equations can be solved as

$$
\begin{aligned}
\widetilde{V}_{1} & =\left(\frac{2 \cos ^{2} \varphi}{\widetilde{T}}-\sqrt{\frac{4 \cos ^{4} \varphi}{\widetilde{T}^{2}}-\frac{\sqrt{2} \cos \varphi}{\widetilde{T}}}\right)^{3}, \\
\widetilde{V}_{2} & =\left(\frac{2 \cos ^{2} \varphi}{\widetilde{T}}+\sqrt{\frac{4 \cos ^{4} \varphi}{\widetilde{T}^{2}}-\frac{\sqrt{2} \cos \varphi}{\widetilde{T}}}\right)^{3}, \\
\varphi & =\frac{\pi-\theta}{3}, \\
\cos \theta & =\frac{\sqrt{2}}{2} \widetilde{T} \\
\widetilde{P}^{*} & =\left(4 \cos \frac{\theta}{3} \cos \frac{\pi+\theta}{3}\right)^{2} \\
& =\left(1-2 \cos \frac{\arccos \left(1-\widetilde{T}^{2}\right)+\pi}{3}\right)^{2} .
\end{aligned}
$$

The last equation $\widetilde{P}^{*}(\widetilde{T})$ is the rescaled phase transition coexistence curve, and it can be rewritten as

$$
\widetilde{T}=\sqrt{\frac{\widetilde{P}^{*}\left(3-\sqrt{\widetilde{P}^{*}}\right)}{2}},
$$

which is exactly the same as (23). So the phase transition coexistence curves obtained by applying the Maxwell equal area law in $T-S$ graph and in $P-V$ graph are consistent with each other.

\section{Swallow Tail Behavior in $G-T$ and $G-P$ Graphs: Phase Transition Coexistence Curve}

In Section 2, we see that the black hole mass can be interpreted as enthalpy. Thus the Gibbs free energy is

$$
G=M-T S,
$$

and its differential form in canonical ensemble can be obtained from (9)

$$
d G=-S d T+\omega d \epsilon+V d P+\Phi d Q=-S d T+V d P
$$

which denotes that the Gibbs free energy is a function of temperature and pressure.

Substituting black hole mass, temperature, and entropy into (32), then making a rescaling by the quantities at the critical point, we will obtain

$$
\begin{aligned}
G & =\frac{\epsilon}{16 \pi} r_{+}-\frac{2 \pi}{3} P r_{+}^{3}+\frac{3 Q^{2}}{4 r_{+}}=\frac{3+6 \widetilde{r}^{2}-\widetilde{P} \widetilde{r}^{4}}{8 \widetilde{r}} \sqrt{\frac{\epsilon Q^{2}}{6 \pi}} \\
& =\widetilde{G} G_{c} \\
T & =\frac{\epsilon}{16 \pi^{2} r_{+}}-\frac{Q^{2}}{4 \pi r_{+}^{3}}+2 P r_{+} \\
& =\frac{3 \widetilde{P} \widetilde{r}^{4}+6 \widetilde{r}^{2}-1}{8 \widetilde{r}^{3}} \frac{\epsilon^{3 / 2}}{24 \sqrt{6} \pi^{5 / 2} Q}=\widetilde{T} T_{c}
\end{aligned}
$$

From the above equation, we can plot $\widetilde{G}-\widetilde{T}$ curves for different $\widetilde{P}$ and plot $\widetilde{G}-\widetilde{P}$ curves for different $\widetilde{T}$ in Figure 5 . One can see that both panels display var der Waals system's swallow tail behavior when $\widetilde{P} \leq 1.0$ and $\widetilde{T} \leq 1.0$. Note that $\widetilde{G}(\widetilde{T})$ does not depend on the topological charge or the electric charge. But by applying a backward rescale, one can find that the swallow tail curves of $G(T)$ in (34) for different $\epsilon$ and $Q$ have conformal symmetry. Since phase transition takes place where the system's two phases have equal Gibbs free energy, temperature, and pressure, the swallow tail's intersection point is exactly the phase transition point. As a result, left panel and right panel have a duality relation and they are equal to each other. Then we will only analyse $\widetilde{G}-\widetilde{T}$ graph to derive the phase transition coexistence curve as follows.

In $\widetilde{G}-\widetilde{T}$ graph, at the phase transition point $\left(\widetilde{T}^{*}, \widetilde{G}^{*}\right)$ for fixed $\widetilde{P}$, we assume that the black hole radius is $\widetilde{r}_{1}$ for one 

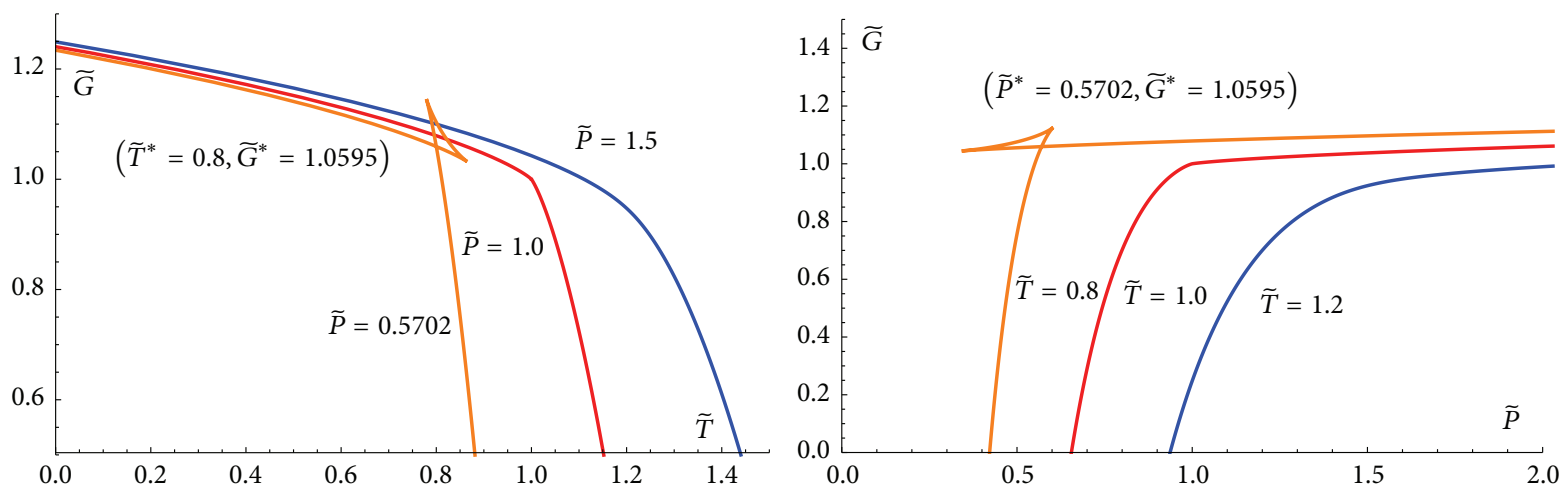

Figure 5: Left panel shows $\widetilde{G}$ vs. $\widetilde{T}$ for different $\widetilde{P}=0.5702,1.0,1.5$. When $\widetilde{P} \leq 1.0$, there is var der Waals system's swallow tail behavior, and the cross point is the phase transition point. Right panel shows $\widetilde{G}$ vs. $\widetilde{P}$ for different $\widetilde{T}=0.8,1.0,1.2$. When $\widetilde{T} \leq 1.0$, there is also var der Waals system's swallow tail behavior, and the cross point is the phase transition point.

phase and $\widetilde{r}_{2}>\widetilde{r}_{1}$ for the other phase. Thus we will have the following equations from (34):

$$
\begin{aligned}
& \widetilde{G}^{*}=\frac{3+6 \widetilde{r}_{1}^{2}-\widetilde{P} \widetilde{r}_{1}^{4}}{8 \widetilde{r}_{1}}=\frac{3+6 \widetilde{r}_{2}^{2}-\widetilde{P}_{2}^{4}}{8 \widetilde{r}_{2}}, \\
& \widetilde{T}^{*}=\frac{3 \widetilde{P} \widetilde{r}_{1}^{4}+6 \widetilde{r}_{1}^{2}-1}{8 \widetilde{r}_{1}^{3}}=\frac{3 \widetilde{P}_{2}^{4}+6 \widetilde{r}_{2}^{2}-1}{8 \widetilde{r}_{2}^{3}} .
\end{aligned}
$$

The above equations can be solved as

$$
\begin{aligned}
& \widetilde{r}_{1}=\frac{\sqrt{3-\sqrt{\widetilde{P}}}-\sqrt{3-3 \sqrt{\widetilde{P}}}}{\sqrt{2 \widetilde{P}}}, \\
& \widetilde{r}_{2}=\frac{\sqrt{3-\sqrt{\widetilde{P}}}+\sqrt{3-3 \sqrt{\widetilde{P}}}}{\sqrt{2 \widetilde{P}}}, \\
& \widetilde{G}^{*}=\frac{\sqrt{6-2 \sqrt{\widetilde{P}}}}{2}, \\
& \widetilde{T}^{*}=\sqrt{\frac{\widetilde{P}(3-\sqrt{\widetilde{P}})}{2}} .
\end{aligned}
$$

The last equation is exactly the same as (23). So the phase transition coexistence curves obtained by analysing the Gibbs free energy in $G-T$ graph and $G-P$ graph, or by applying the Maxwell equal area law in $T-S$ graph and $P-V$ graph, are consistent with each other.

\section{Conclusion and Discussion}

Treating the cosmological constant as a variable $[13,14]$ and the spatial curvature as topological charge [58, 59], thermodynamics of electrically charged Reissner-Nordström AdS black holes are investigated. Firstly, by variation of the equipotential equation on horizon, the extended thermodynamic first law is obtained. From the extended first law, a conjugate potential correspondent to the topological charge is arisen. Meanwhile, if the black hole volume is defined as $V=$
$\left(\Omega_{d-2} /(d-1)\right) r_{+}^{d-1}$, then its conjugate pressure is naturally assigned as the cosmological constant and the black hole mass as enthalpy.

Secondly, in four dimensional space-time and canonical ensemble with fixed electric charge and topological charge, the isobaric specific heat $C_{P}$ is calculated and the corresponding divergent solutions are derived. The two solutions merge into one at the critical point with $P_{c}=\epsilon^{2} /\left(1536 \pi^{3} Q^{2}\right), r_{c}=$ $2 \sqrt{6 \pi / \epsilon} Q$. When $P<P_{c}$, the curve of specific heat has two divergent points and is divided into three regions. The specific heat is positive for both the large radius region and the small radius region which are thermodynamically stable, while it is negative for the medium radius region which is unstable. When $P>P_{c}$, the specific heat is always positive implying the black holes are stable and no phase transition will take place.

Thirdly, rescaling the quantities by those at the critical point, the behavior of temperature in $\widetilde{T}-\widetilde{S}$ graph and the behavior of pressure in $\widetilde{P}-\widetilde{V}$ graph are studied. They exhibit the interesting van de Waals gas-liquid system's behavior. When $\widetilde{P}>1, \widetilde{T}>1$, the curves vary monotonically and no phase transition will take place. When $\widetilde{P}<1, \widetilde{T}<$ 1 , the curves display an oscillatory behavior which signals phase transition. The oscillatory part is replaced by an isobar according to the Maxwell's equal area law and the analytical phase transition coexistence curves (rescaled) are obtained which are consistent with each other. Then by making a backward rescale, the explicit phase transition coexistence curve is derive in (24) and the phase diagrams are shown in Figure 3.

Fourthly, van der Waals system's swallow tail behavior is observed in the $\widetilde{G}-\widetilde{T}$ graph and $\widetilde{G}-\widetilde{P}$ graph when $\widetilde{P}<1, \widetilde{T}<$ 1. The swallow tail's intersection point is the phase transition point. By analytically solving the constraint equations, the rescaled phase transition coexistence curve is obtained which is consistent with those derived in $\widetilde{T}-\widetilde{S}$ graph and $\widetilde{P}-\widetilde{V}$ graph.

From the above detailed study in canonical ensemble, the analogy of RN-AdS black hole as van der Waals system has been examined when the spatial curvature is treated as topological charge and the cosmological constant is treated 
as pressure. Both the systems share the same oscillatory behavior and swallow tail behavior. Comparing with the case when the spatial curvature is fixed $[14,15]$, our phase transition diagram in Figure 3 is four dimensional $(T, P, Q, \epsilon)$, which is more rich with an extra parameter, the topological charge.

A further investigation in grand canonical ensemble is outside the scope of this paper, but it is surely a very interesting direction for future research. The influence of this topological charge on black hole thermodynamics in other gravity theories (such as the Lovelock, Gauss-Bonnet theory, and $\mathrm{f}(\mathrm{R})$ theory) and different dimensional spacetime also deserves to be disclosed in the future research. Another interesting future research line is the comparison of the influence between the electric charge and the topological charge.

In the end, we would like to point out that the black hole thermodynamics discussed in this paper is based on the first law derived from the equipotential surface $f(r)=c$ with $c=0$. A more conventional way to compute thermodynamics quantities is the Euclidean formalism, where the free energy is computed firstly and then the other quantities follow from it. The difference between these two formalisms remains unknown which deserves to be investigated in future.

\section{Data Availability}

The data used to support the findings of this study are included within the article.

\section{Conflicts of Interest}

The author declares that they have no conflicts of interest.

\section{Acknowledgments}

This research is supported by Department of Education of Guangdong Province, China (Grant no. 2017KQNCX124).

\section{References}

[1] J. D. Bekenstein, "Black holes and entropy," Physical Review D: Particles, Fields, Gravitation and Cosmology, vol. 7, pp. 23332346, 1973.

[2] J. M. Bardeen, B. Carter, and S. W. Hawking, "The four laws of black hole mechanics," Communications in Mathematical Physics, vol. 31, pp. 161-170, 1973.

[3] S. W. Hawking, "Particle creation by black holes," Communications in Mathematical Physics, vol. 43, no. 3, pp. 199-220, 1975.

[4] S. W. Hawking and D. N. Page, "Thermodynamics of black holes in anti-de Sitter space," Communications in Mathematical Physics, vol. 87, no. 4, pp. 577-588, 1982/83.

[5] J. Maldacena, "The large $N$ limit of superconformal field theories and supergravity," Advances in Theoretical and Mathematical Physics, vol. 2, no. 2, pp. 231-252, 1998.

[6] S. S. Gubser, I. R. Klebanov, and A. M. Polyakov, "Gauge theory correlators from non-critical string theory," Physics Letters B, vol. 428, no. 1-2, pp. 105-114, 1998.
[7] E. Witten, "Anti de Sitter space and holography," Advances in Theoretical and Mathematical Physics, vol. 2, no. 2, pp. 253-291, 1998.

[8] E. Witten, "Anti-de Sitter space, thermal phase transition, and confinement in gauge theories," Advances in Theoretical and Mathematical Physics, vol. 2, no. 3, pp. 505-532, 1998.

[9] A. Chamblin, R. Emparan, C. V. Johnson, and R. C. Myers, "Charged AdS black holes and catastrophic holography," Physical Review D: Particles, Fields, Gravitation and Cosmology, vol. 60, no. 6, 1999.

[10] R. Banerjee and D. Roychowdhury, "Thermodynamics of phase transition in higher dimensional AdS black holes," Journal of High Energy Physics, vol. 2011, article 4, 2011.

[11] R. Banerjee, S. K. Modak, and D. Roychowdhury, "A unified picture of phase transition: From liquid-vapour systems to AdS black holes," Journal of High Energy Physics, vol. 2012, no. 10, 2012.

[12] A. Chamblin, R. Emparan, C. V. Johnson, and R. C. Myers, "Holography, thermodynamics, and fluctuations of charged AdS black holes," Physical Review D: Particles, Fields, Gravitation and Cosmology, vol. 60, no. 10, Article ID 104026, 1999.

[13] D. Kastor, S. Ray, and J. Traschen, "Enthalpy and the mechanics of AdS black holes," Classical and Quantum Gravity, vol. 26, no. 19, Article ID 195011, 2009.

[14] D. Kubiznak and R. B. Mann, "P-V criticality of charged AdS black holes," Journal of High Energy Physics, vol. 2012, no. 7, article 033, 2012.

[15] E. Spallucci and A. Smailagic, "Maxwell's equal-area law for charged Anti-de Sitter black holes," Physics Letters B, vol. 723, no. 4-5, pp. 436-441, 2013.

[16] S. Gunasekaran, D. Kubiznak, and R. B. Mann, "Extended phase space thermodynamics for charged and rotating black holes and Born-Infeld vacuum polarization," Journal of High Energy Physics, vol. 2012, no. 11, article no. 110, 2012.

[17] S. H. Hendi and M. H. Vahidinia, "Extended phase space thermodynamics and $P-V$ criticality of black holes with a nonlinear source," Physical Review D: Particles, Fields, Gravitation and Cosmology, vol. 88, no. 8, Article ID 084045, pp. 1-11, 2013.

[18] S. Chen, X. Liu, C. Liu, and J. Jing, " $P-V$ criticality of AdS black hole in $f(R)$ gravity," Chinese Physics Letters, vol. 30, no. 6, Article ID 060401, 2013.

[19] R. Zhao, H. H. Zhao, M. S. Ma, and L. C. Zhang, "On the critical phenomena and thermodynamics of charged topological dilaton AdS black holes," The European Physical Journal C, vol. 73, no. 12, pp. 1-10, 2013.

[20] N. Altamirano, D. Kubiznak, and R. Mann, "Reentrant phase transitions in rotating AdS black holes," Physical Review D: Particles, Fields, Gravitation and Cosmology, vol. 88, no. 10, Article ID 101502, 2013.

[21] R.-G. Cai, L.-M. Cao, L. Li, and R.-Q. Yang, "P-V criticality in the extended phase space of Gauss-Bonnet black holes in AdS space," Journal of High Energy Physics, vol. 2013, no. 9, article no. 5, 2013.

[22] N. Altamirano, D. Kubizňák, R. B. Mann, and Z. Sherkatghanad, "Kerr-AdS analogue of triple point and solid/liquid/gas phase transition," Classical and Quantum Gravity, vol. 31, no. 4, Article ID 042001, 2014.

[23] W. Xu, H. Xu, and L. Zhao, "Gauss-bonnet coupling constant as a free thermodynamical variable and the associated criticality," The European Physical Journal C, vol. 74, no. 7, article no. 2970, 2014. 
[24] J.-X. Mo and W.-B. Liu, "Ehrenfest scheme for $P-V$ criticality in the extended phase space of black holes," Physics Letters B, vol. 727, no. 1-3, pp. 336-339, 2013.

[25] D.-C. Zou, S.-J. Zhang, and B. Wang, "Critical behavior of BornInfeld AdS black holes in the extended phase space thermodynamics," Physical Review D: Particles, Fields, Gravitation and Cosmology, vol. 89, Article ID 044002, 2014.

[26] J.-X. Mo and W.-B. Liu, " $P-V$ criticality of topological black holes in Lovelock-Born-Infeld gravity," The European Physical Journal C, vol. 74, article 2836, 2014.

[27] N. Altamirano, D. Kubizňák, R. Mann, and Z. Sherkatghanad, "Thermodynamics of rotating black holes and black rings: phase transitions and thermodynamic volume," Galaxies, vol. 2, no. 1, pp. 89-159, 2014.

[28] S.-W. Wei and Y.-X. Liu, "Critical phenomena and thermodynamic geometry of charged Gauss-Bonnet AdS black holes," Physical Review D: Particles, Fields, Gravitation and Cosmology, vol. 87, Article ID 044014, 2013.

[29] S. W. Wei and Y. X. Liu, "Triple points and phase diagrams in the extended phase space of charged Gauss-Bonnet black holes in AdS space," Physical Review D, vol. 90, Article ID 044057, 2014.

[30] L.-C. Zhang, M.-S. Ma, H.-H. Zhao, and R. Zhao, "Thermodynamics of phase transition in higher-dimensional ReissnerNordström-de Sitter black hole," The European Physical Journal C, vol. 74, article no. 3052, 2014.

[31] J.-X. Mo and W.-B. Liu, "Ehrenfest scheme for P-V criticality of higher dimensional charged black holes, rotating black holes, and Gauss-Bonnet AdS black holes," Physical Review D: Particles, Fields, Gravitation and Cosmology, vol. 89, no. 8, Article ID 084057, 2014.

[32] D. C. Zou, Y. Liu, and B. Wang, "Critical behavior of charged Gauss-Bonnet-AdS black holes in the grand canonical ensemble," Physical Review D: Particles, Fields, Gravitation and Cosmology, vol. 90, no. 4, Article ID 044063, 2014.

[33] Z. Zhao and J. Jing, "Ehrenfest scheme for complex thermodynamic systems in full phase space," Journal of High Energy Physics, vol. 2014, no. 11, article no. 37, 2014.

[34] R. Zhao, M. Ma, H. Zhao, and L. Zhang, "The critical phenomena and thermodynamics of the reissner-nordstrom-de sitter black hole," Advances in High Energy Physics, vol. 2014, Article ID 124854, 6 pages, 2014.

[35] H. Xu, W. Xu, and L. Zhao, "Extended phase space thermodynamics for third-order Lovelock black holes in diverse dimensions," The European Physical Journal C, vol. 74, article 3074, 2014.

[36] A. M. Frassino, D. Kubizňák, R. B. Mann, and F. Simovic, "Multiple reentrant phase transitions and triple points in Lovelock thermodynamics," Journal of High Energy Physics, vol. 2014, no. 9, article no. 80, 2014.

[37] J. L. Zhang, R. G. Cai, and H. Yu, "Phase transition and thermodynamical geometry for Schwarzschild AdS black hole in $\mathrm{AdS}_{5} \times \mathrm{S}_{5}$ spacetime," Journal of High Energy Physics, vol. 2015, no. 2, article no. 143, 2015.

[38] B. Mirza and Z. Sherkatghanad, "Phase transitions of hairy black holes in massive gravity and thermodynamic behavior of charged AdS black holes in an extended phase space," Physical Review D: Particles, Fields, Gravitation and Cosmology, vol. 90, no. 8, article no. 084006, 2014.

[39] B. P. Dolan, A. Kostouki, D. Kubizňák, and R. B. Mann, "Isolated critical point from Lovelock gravity," Classical and Quantum Gravity, vol. 31, no. 24, Article ID 242001, 2014.
[40] A. Rajagopal, D. Kubiznak, and R. B. Mann, "Van der Waals black hole," Physics Letters B, vol. 737, pp. 277-279, 2014.

[41] Y. Liu, D.-C. Zou, and B. Wang, "Signature of the Van der Waals like small-large charged AdS black hole phase transition in quasinormal modes," Journal of High Energy Physics, vol. 2014, no. 09, article no. 179, 2014.

[42] S. H. Hendi, R. B. Mann, S. Panahiyan, and B. Eslam Panah, "Van der Waals like behavior of topological AdS black holes in massive gravity," Physical Review D: Particles, Fields, Gravitation and Cosmology, vol. 95, no. 2, Article ID 021501, 2017.

[43] S. H. Hendi, B. Eslam Panah, and S. Panahiyan, "Topological charged black holes in massive gravity's rainbow and their thermodynamical analysis through various approaches," Physics Letters B, vol. 769, pp. 191-201, 2017.

[44] S.-Q. Lan, J.-X. Mo, and W.-B. Liu, "A note on Maxwell's equal area law for black hole phase transition," The European Physical Journal C, vol. 75, no. 9, article no. 419, 2015.

[45] M.-S. Ma and R.-H. Wang, "Peculiar P-V criticality of topological Horava-Lifshitz black holes," Physical Review D: Particles, Fields, Gravitation and Cosmology, vol. 96, no. 2, article no. 024052, 2017.

[46] S. W. Wei, B. Liang, and Y. Liu, "Critical phenomena and chemical potential of a charged AdS black hole," Physical Review D: Particles, Fields, Gravitation and Cosmology, vol. 96, no. 12, article no. 124018, 2017.

[47] K. Bhattacharya and B. R. Majhi, “Thermogeometric description of the van der Waals like phase transition in AdS black holes," Physical Review D: Particles, Fields, Gravitation and Cosmology, vol. 95, no. 10, article no. 104024, 2017.

[48] S. H. Hendi, B. E. Panah, S. Panahiyan, and M. S. Talezadeh, "Geometrical thermodynamics and P-V criticality of the black holes with power-law Maxwell field," The European Physical Journal C, vol. 77, no. 2, article no. 133, 2017.

[49] X. M. Kuang and O. Miskovic, "Thermal phase transitions of dimensionally continued AdS black holes," Physical Review D: Particles, Fields, Gravitation and Cosmology, vol. 95, no. 4, Article ID 046009, 2017.

[50] S. Fernando, "P-V criticality in AdS black holes of massive gravity," Physical Review D: Particles, Fields, Gravitation and Cosmology, vol. 94, no. 12, Article ID 124049, 2016.

[51] B. R. Majhi and S. Samanta, "P-V criticality of AdS black holes in a general framework," Physics Letters B, vol. 773, pp. 203-207, 2017.

[52] S. He, L. F. Li, and X. X. Zeng, "Holographic Van der Waals-like phase transition in the Gauss-Bonnet gravity," Nuclear Physics $B$, vol. 915, p. 243, 2017.

[53] J. Sadeghi, B. Pourhassan, and M. Rostami, "P-V criticality of logarithm-corrected dyonic charged AdS black holes," Physical Review D: Particles, Fields, Gravitation and Cosmology, vol. 94, no. 6, Article ID 064006, 2016.

[54] X. X. Zeng and L. F. Li, "Van der waals phase transition in the framework of holography," Physics Letters B, vol. 764, article no. 100, 2017.

[55] P. H. Nguyen, "An equal area law for holographic entanglement entropy of the AdS-RN black hole," Journal of High Energy Physics, vol. 2015, no. 12, article no. 139, 2015.

[56] J. F. Xu, L.-M. Cao, and Y.-P. Hu, "P-V criticality in the extended phase space of black holes in massive gravity," Physical Review D: Particles, Fields, Gravitation and Cosmology, vol. 91, no. 12, Article ID 124033, 2015. 
[57] Z.-Y. Nie and H. Zeng, "P-T phase diagram of a holographic $\mathrm{s}+\mathrm{p}$ model from Gauss-Bonnet gravity," Journal of High Energy Physics, no. 10, article no. 047, 2015.

[58] Y. Tian, X.-N. Wu, and H. Zhang, "Holographic entropy production," Journal of High Energy Physics, no. 10, article no. 170, 2014.

[59] Y. Tian, “The last (lost) charge of a black hole," https://arxiv.org/ abs/1804.00249, 2018.

[60] S. Q. Lan, G. Q. Li, J. X. Mo, and X. B. Xu, "New phase transition related to the the black hole's topological charge," https://arxiv.org/abs/1804.06652, 2018. 

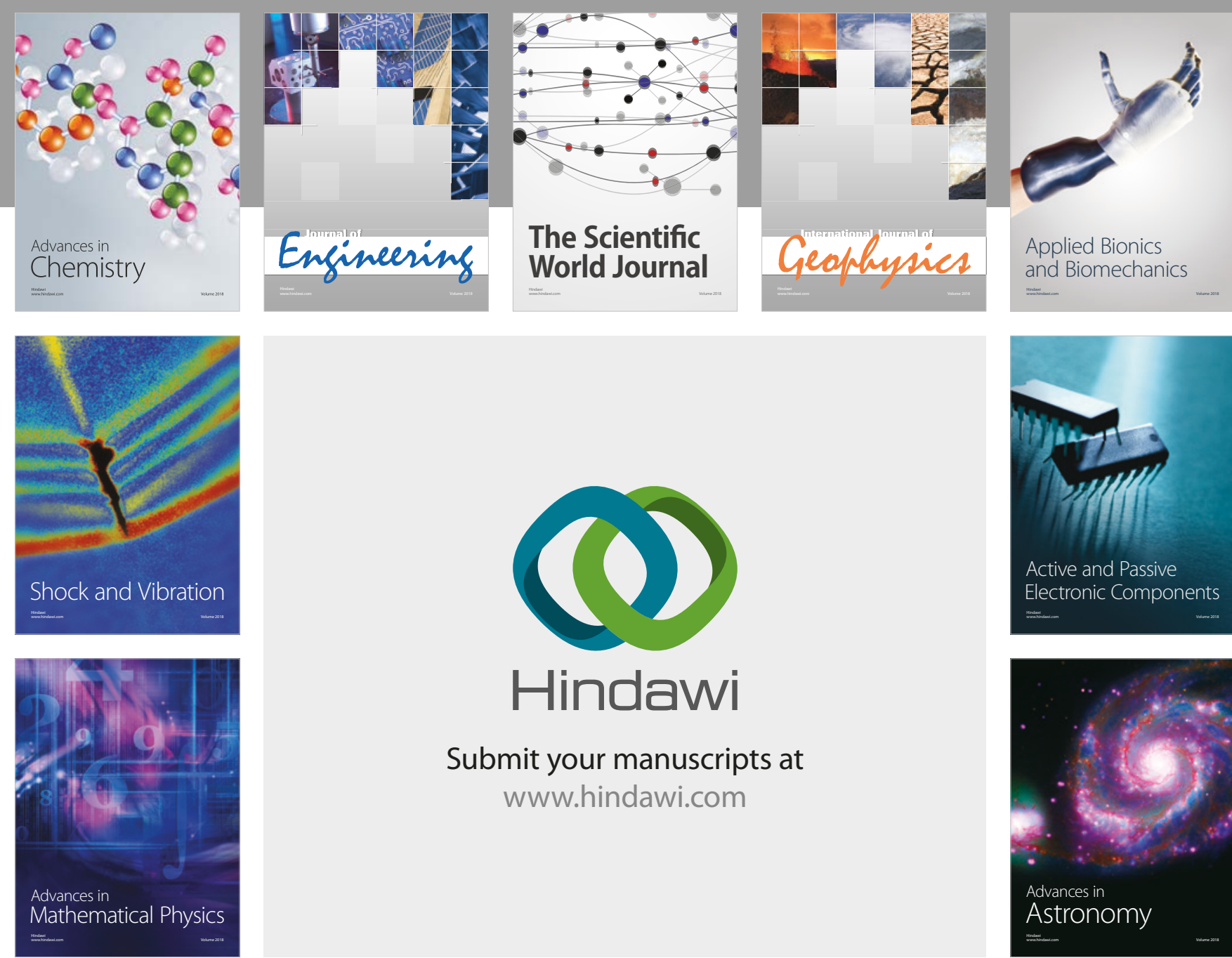

Submit your manuscripts at

www.hindawi.com

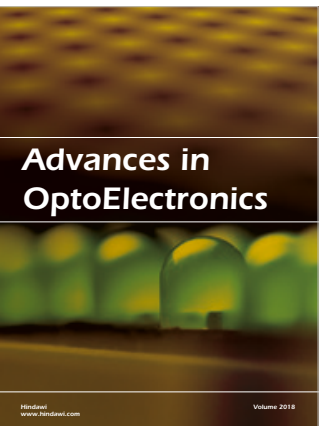

\section{Rotcting Machinery}
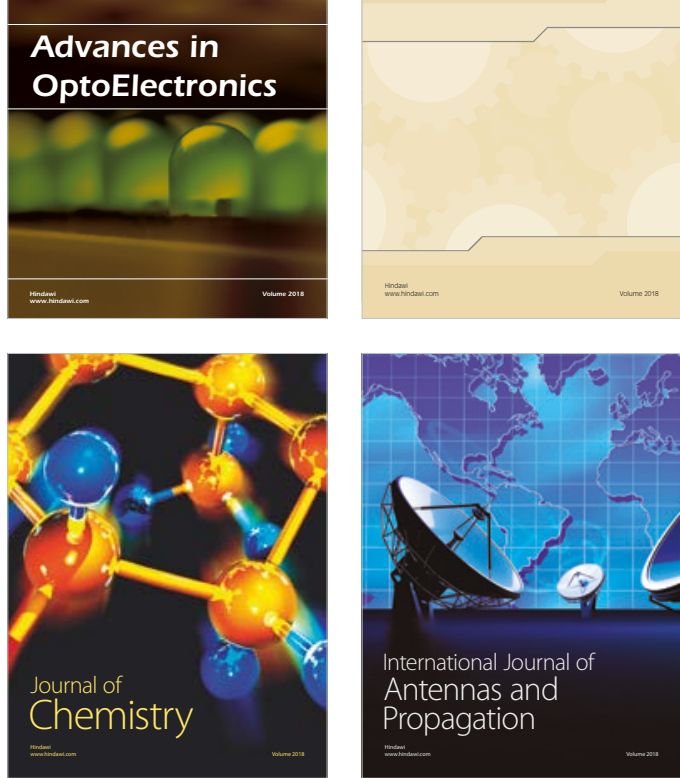

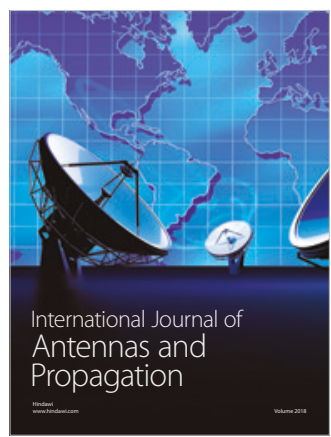

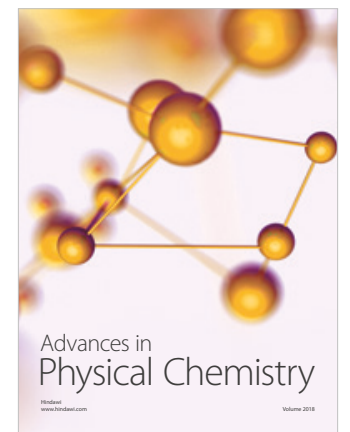

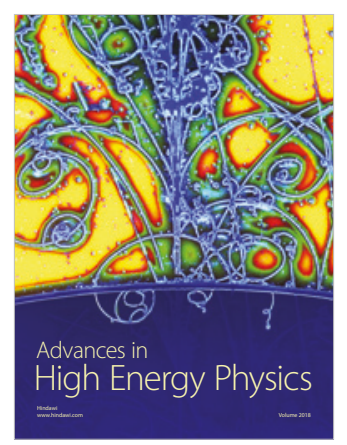

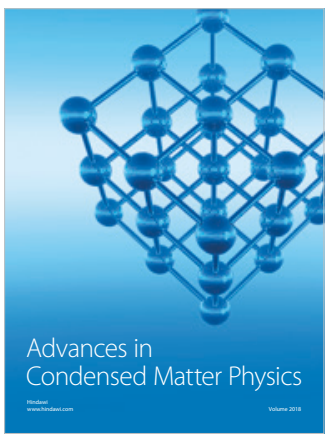

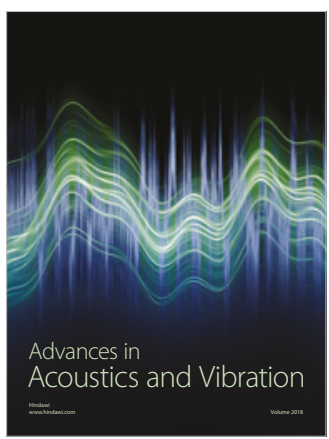

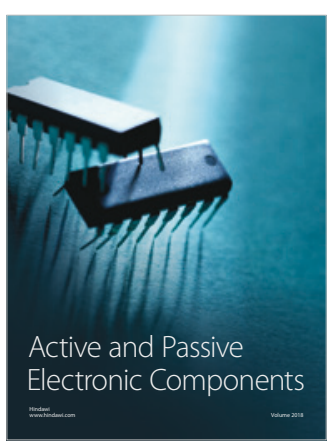
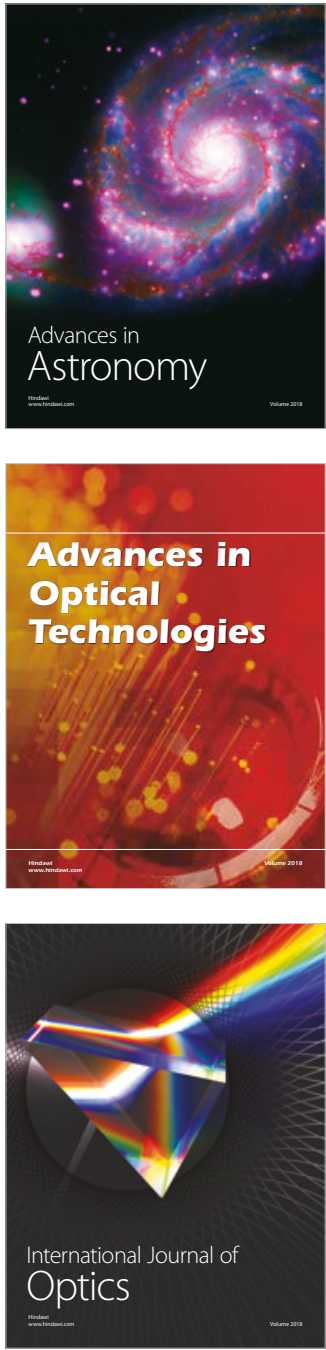